\title{
Hepatitis A Virus in Lao People's Democratic Republic: Seroprevalence and Risk Factors
}

\author{
Vilaysone Khounvisith, ${ }^{1} \dagger$ Xaipasong Xaiyaphet, ${ }^{2} \dagger$ Phetsavanh Chanthavilay, ${ }^{2}$ Phonethipsavanh Nouanthong, ${ }^{1}$ \\ Bounta Vongphachanh, ${ }^{1}$ Daniel Reinharz, ${ }^{2}$ Claude P. Muller, ${ }^{1,3}$ and Antony P. Black ${ }^{1 *}$ \\ ${ }^{1}$ Lao-Lux Laboratory, Institut Pasteur du Laos, Vientiane, Lao PDR; ${ }^{2}$ Institut de la Francophonie pour la Medecine Tropicale, Lao PDR; ${ }^{3}$ Department \\ of Infection and Immunity, Luxembourg Institute of Health, Esch-sur-Alzette, Luxembourg
}

\begin{abstract}
Despite several recent reports of outbreaks of hepatitis A, little is known about the disease burden in Lao People's Democratic Republic (PDR). We conducted a cross-sectional age-stratified seroprevalence study of antihepatitis A virus (HAV) IgG and risk factors in a rural province (Xiengkhouang) and Vientiane capital in Lao PDR. Overall, $62 \%$ of participants were anti-HAV positive in Xiengkhouang Province compared with $45.5 \%$ in Vientiane capital. In Xiengkhouang, $23.7 \%$ of 5 - to 10 -year-olds were already seropositive compared with $5 \%$ in Vientiane. A dramatic increase in seroprevalence occurred between 15- to 20-year and 21- to 30-year age-groups (35.7-62.4\%, Xiengkhouang, and $11.5-69.7 \%$, Vientiane) until essentially all older adults were positive in both locations. The main risk factors for HAV antibodies were age, non-Lao-Tai ethnicity, and food-related risk factors. In conclusion, Lao children seem to be exposed very early to HAV, particularly in rural settings, and exposure continues throughout their lives, mostly without being reported. In the older birth cohorts, the high seroprevalence may largely reflect poor sanitation and exposure during childhood. In Vientiane capital, the lower seroprevalence at young ages may reflect better water sanitation since the late 1990s. A comparison with neighboring Thailand indicates that the impact of improved sanitation in Lao PDR began only two decades later. Further improvements in water sanitation, in particular in the rural districts, and better food hygiene are warranted. Our study also suggests that HAV infections are underreported. Improved reporting would provide guidance for targeted interventions to further reduce HAV infections.
\end{abstract}

\section{INTRODUCTION}

Hepatitis A virus (HAV) infection is a global public health problem, with approximately 1.5 million symptomatic cases annually and tens of millions of asymptomatic infections. ${ }^{1,2}$ After an incubation period of 15-50 days, clinical symptoms vastly differ in severity. Whereas most children younger than 6 years are asymptomatic, older children and adults have symptoms in up to $70 \%$ of cases. ${ }^{1}$ Approximately $70 \%$ of adults with acute HAV infection have jaundice, fever, hepatomegaly, tenderness, nausea, vomiting, abdominal pain, fatigue, and malaise., ${ }^{3,4}$ The diagnosis of acute infection is by serology or virus detection in the blood or stool by reverse transcription polymerase chain reaction. ${ }^{5}$ Hospitalization may be required for severe cases, but there is no specific treatment. Following infection, anti-HAV antibodies provide lifelong immunity against all strains. Hepatitis A vaccines are available for individuals aged 1-2 years, with an efficacy of 97-100\%. ${ }^{6}$ Antibodies may persist for more than 20 years after complete vaccination. ${ }^{7}$

Hepatitis $A$ is endemic in regions with poor sanitation, and outbreaks are often due to contaminated food. The incidence of HAV infections varies worldwide and by age. .,8 $^{3,8}$

In many countries in eastern Europe, Africa, Asia, and South America, depending on sanitation, there is a high incidence of infection during childhood and adolescence. In some countries, $90 \%$ of children are infected by age 10 years (usually asymptomatic). In developed countries with better hygiene, such as North America, western Europe, Australia, and Japan, infection rates in children are lower, with a shift of the burden of infection to adolescents and young adults. ${ }^{3,9}$

In Lao People's Democratic Republic (PDR), more than 1,000 cases of acute jaundice syndrome were reported in

\footnotetext{
*Address correspondence to Antony P. Black, Lao-Lux Laboratory, Institut Pasteur du Laos, PO Box 3560, Rue Samsenthai, Vientiane, Lao PDR. E-mail: a.black@pasteur.la

†These authors contributed equally to this work.
}

2016, most of them from the north-eastern province of Xiengkhouang, Peak district, and outbreaks have continued since in the same province. Hepatitis A virus outbreaks have also been confirmed in other parts of the country, such Vientiane capital and Vientiane Province (approximately 900 cases in 2017) (Amphaiy Khamsing, National Surveillance, Risk assessment and Response, personal communication). There is no routine vaccination for HAV in Lao PDR, and the vaccine is not readily available.

Despite these few reported outbreaks of HAV in the country, little is known about the epidemiology of the disease and specific risk factors in Lao PDR. The aim of this study was to determine the age-specific HAV seroprevalence in Xiengkhouang Province and in Vientiane capital and to describe risk behaviors in this population.

\section{MATERIALS AND METHODS}

Recruiting in Xiengkhouang Province. This crosssectional study was conducted between March 21, 2017 and June 02, 2017 in two districts of Xiengkhouang Province (Phasay and Peak), Lao PDR, a country with a population of seven million. Xiengkhouang Province includes eight districts, with a population of around 270,000 (in 2010), mostly of the Phouthay ethnic group (Lao-Tai family). ${ }^{10}$ Peak and Phasay were selected because of reports of recent HAV outbreaks within these two districts.

Age-stratified sample size sampling was carried out based on five age-groups: $5-10,11-20,21-30,31-40$, and $>40$ years. The population size for each age-group was obtained from the Lao National Statistics Bureau. The sample size was thereafter adjusted to take into consideration the design effect by multiplying the calculated sample size by a correlation coefficient of 1.5 as a result of the expected variance in clustering sampling and by adding $10 \%$ to compensate for refusal or losses. Therefore, the total sample size was 400 people. Proportion size allocation was applied to determine the total 
number of samples required by age-group. This number was divided by the number of selected villages $(20)$ to obtain the number of individuals needed to enroll per village. ${ }^{11}$ Two-stage sampling was applied to select villages and individuals included in the study. Twenty villages (minimum number needed for a cluster design) were selected as follows; villages were stratified into two strata: either urban/semi-urban (Peak district) or rural (Phasay district). Using a "proportion-to-size" allocation approach, 13 villages were randomly selected among 108 from Peak. Seven of 32 villages were selected from Phasay. Participants from each village were randomly selected from the household list obtained from the head of the village.

Interviews were held in the village office, in a school, or in a temple nearby. The researcher explained the aim of the study to the participants and/or their parents/guardians. For participants younger than 18 years, assent was taken in addition to informed consent from the parents/guardians. Questionnaires were completed by the parents/guardians. The questionnaire was based on the conceptual framework and consisted of four sections: sociodemographic factors, socioeconomic factors, behavior and lifestyle, and past history. This study was approved by the Lao National Ethics Committee for Health Research (NEHCR 034/2017).

Recruiting in Vientiane capital. Children aged $0-16$ years were recruited while attending a fever rash clinic in the children hospital in 2015 ( $n=200$, ethics approval number NEHCR 070/ 2013). Adolescents aged 11 to 20 years were recruited during a school-based study in 2018 ( $n=200$, ethics approval number NEHCR 022/2018). Adult healthcare workers aged 20 to 50 years were recruited from central, district, and health centers between 2013 and 2016 ( $n=140$, ethics approval number NEHCR 069/2013). Blood donors were recruited between 2013 and 2015 ( $n=255$, ethics approval number NEHCR 059/2013). Both healthcare workers and blood donors were specifically included in the older age-group because of availability of the serum bank from these populations in Vientiane capital. However, these may not be representative of the general population.

Serology. Five milliliters of blood was collected from each participant. Serum was separated by centrifugation in the laboratory of the provincial hospital and was stored at $-20^{\circ} \mathrm{C}$. Serum was subsequently transported in a cool box to the Institute Pasteur du Laos in Vientiane, where it was stored at $-80^{\circ} \mathrm{C}$. Anti-HAV IgG was measured using a competitive ELISA (Diasorin, Sallugia, Italy). According to the manufacturer's instructions, quantification of antibody titers greater than $20 \mathrm{mIU} / \mathrm{mL}$ confers immunity to HAV and the ELISA has 99\% sensitivity and $99.8 \%$ specificity. Results were classified as positive, negative, or borderline. Borderline samples were grouped with the negative samples for analysis. As there is no routine vaccination for HAV in Lao PDR, anti-HAV IgG antibodies are indicative of previous exposure in this context.

Statistical analysis. Cross-sectional data were double entered in Epidata version 3.1 and analyzed with STATA software version 12 (StataCorp LLC, College Station, TX). Initially, descriptive analyses were made on all variables. Bivariate analysis was used to test the association between antiHAV positivity and independent variables. The chi-square test or Fisher's exact test was used when appropriate. Student's $t$ test and analysis of variance compared the levels of anti-HAV titer among two or more than two groups, respectively. Moreover, the correlations between continuous variables were checked using Pearson's correlation test with $P$-value less than 0.2. Multivariate analysis variables were introduced into the final model of linear regression. For the multivariate analysis, we divided the source of water into two groups: plastic water and "other". The result was reported as odds ratio $(\mathrm{OR})$ with $95 \% \mathrm{Cl}$ and $P$-value.

\section{RESULTS}

Demographic characteristics and risk factors in Xiengkhouang Province. Two hundred sixty serum samples were collected in Peak and 140 in Phasay district. Fifty-nine children were aged 5 to 10 years, 81 adolescents aged 11 to 20 years, and 260 adults aged $>21$ years (mean age was $29.1 \pm$ 16 years). Most participants (81\%) were of Lao-Tai ethnicity.

We found a high level of risk practices in this population. Although most participants reported using bottled water for drinking (65.8\%), a substantial proportion used well water for drinking $(57.5 \%)$ and cooking $(98.2 \%)$; many participants used river water for cooking (66\%). We also investigated a number of food-related risk factors. Raw fish and raw meat were consumed at least once per month by about $20 \%$ of participants. The vast majority $(84 \%)$ of participants ate snails at least once per month, and $46.5 \%$ ate shellfish once per month. Furthermore, although most participants always washed their hands before eating $(76.3 \%)$ or after using the toilet $(71 \%)$, few reported to always use soap to wash hands $(26.5 \%)$.

Seroprevalence of HAV in Xiengkhouang Province. Overall, anti-HAV IgG seropositivity was $62.3 \%$, with $2.8 \%$ $(11 / 40)$ borderline. The seroprevalence by age was similar in both districts: $24 \%$ of the 5- to 10 -year-olds were seropositive and by the age of $>40$, essentially all were seropositive (Figure 1).

Logistical regression was performed on factors that were associated with anti-HAV positivity with $P<0.2$ in the bivariate analysis (Table 1). After multivariate analysis, four factors were significantly associated with past exposure: age greater than 20 years (OR 12.7 [7.4-21.8]), being of non-Lao-Tai ethnicity (OR 6.6 [3.1-14.4]), eating snails (OR 13.5 [2.4-77.4]), and washing hands with soap (OR 2.5 [1.4-4.3]).

Subgroup analysis by age revealed the following risk factors in children and adolescents younger than 20 years: non-Lao-Tai ethnicity $(\mathrm{OR}=3.8$ [1.6-10.07]) and eating shellfish (snail)

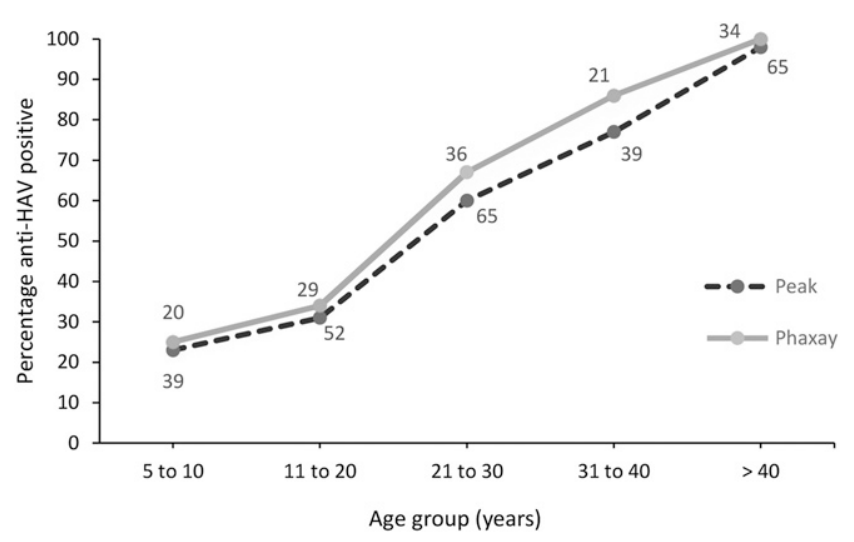

FIGURE 1. Age-specific seroprevalence of antibodies to the HAV (lgG) in Peak and Phasay districts in Xiengkhouang Province. Numbers indicate the total number of participants per age-group. 
TABLE 1

Sociodemographic data and risk factors in Xiengkhouang Province

\begin{tabular}{|c|c|c|c|c|c|c|c|c|}
\hline \multirow[b]{2}{*}{ Variable } & & \multirow{2}{*}{$\begin{array}{c}n \text { positive per } \\
\text { group (\%) }\end{array}$} & \multicolumn{3}{|c|}{ Bivariate analysis } & \multicolumn{3}{|c|}{ Multivariate analysis } \\
\hline & & & OR & $95 \% \mathrm{Cl}$ & $P$-value & OR & $95 \% \mathrm{Cl}$ & $P$-value \\
\hline \multirow[t]{2}{*}{ Age (years) } & $<20$ & 40/140 (29) & Ref & & & Ref & & \\
\hline & $>20$ & 209/260 (80) & 10.2 & [6.3-16.5] & $<0.0001$ & 12.7 & [7.4-21.8] & $<0.0001$ \\
\hline \multirow[t]{2}{*}{ District } & Peak & $158 / 260(61)$ & Ref & & & & & \\
\hline & Phasay & $91 / 140(65)$ & 1.19 & {$[0.78-1.83]$} & 0.405 & & & NS \\
\hline \multirow[t]{2}{*}{ Gender } & Male & $150 / 247(61)$ & Ref & & & & & \\
\hline & Female & 99/153 (65) & 0.84 & {$[0.55-1.28]$} & 0.425 & & & NS \\
\hline \multirow{2}{*}{ Ethnicity } & Lao-Tai & $187 / 324(58)$ & Ref & & & Ref & & \\
\hline & Non-Lao-Tai & 62/76 (82) & 3.24 & [1.74-6.03] & 0.0001 & 6.6 & [3.1-14.4] & $<0.0001$ \\
\hline \multirow[t]{2}{*}{ Religion } & Buddhism & $197 / 337(58)$ & Ref & & & & & \\
\hline & Animism & $52 / 63(83)$ & 3.35 & {$[1.69-6.66]$} & 0.001 & & & NS \\
\hline \multirow[t]{2}{*}{ Household member } & $<5$ & $152 / 247(62)$ & Ref & & & & & \\
\hline & $>5$ & $97 / 153(63)$ & 1.08 & {$[0.71-1.64]$} & 0.709 & & & NS \\
\hline \multirow[t]{2}{*}{ Level of education completed } & Lower secondary school & $99 / 155(64)$ & Ref & & & & & \\
\hline & Upper secondary school & $150 / 245(61)$ & 0.89 & {$[0.5-1.3]$} & 0.594 & & & NS \\
\hline \multirow[t]{2}{*}{ Occupation } & Farmer & $182 / 226(81)$ & Ref & & & & & \\
\hline & Non-farmer & 67/174 (39) & 1.15 & {$[0.09-0.23]$} & $<0.0001$ & & & NS \\
\hline \multirow[t]{2}{*}{ Salary income per month (Lao Kip) } & $<3 \mathrm{M}$ & $209 / 337(62)$ & Ref & & & & & \\
\hline & $>3 \mathrm{M}$ & $40 / 63(63)$ & 1.06 & {$[0.6-1.8]$} & 0.82 & & & NS \\
\hline \multirow[t]{6}{*}{ Source of drinking water } & Tap water & $0 / 1(0)$ & 1 & & & & & \\
\hline & Boiled water & $126 / 198(64)$ & 1.12 & {$[0.7-1.6]$} & $0 . \overline{5} 71$ & & & \\
\hline & Natural water (river) & $6 / 9(67)$ & 1.12 & [0.3-4.9] & 0.783 & & & \\
\hline & Plastic bottle waters & $147 / 259(57)$ & 0.5 & {$[0.3-0.7]$} & 0.002 & & & \\
\hline & Drilled water & $9 / 13(69)$ & 1.37 & [0.4-4.5] & 0.599 & & & \\
\hline & Other & $50 / 73(68)$ & & & & & & NS \\
\hline \multirow{5}{*}{$\begin{array}{l}\text { Source of water (apart from } \\
\text { drinking) }\end{array}$} & Tap water & $9 / 12(4)$ & 1.85 & {$[0.4-\overline{6} .9]$} & $0 . \overline{3} 62$ & & & \\
\hline & Boiled water & $0 / 0(0)$ & & 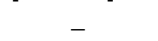 & - & & & \\
\hline & Natural water (river) & $158 / 264(63)$ & 1 & & & & & \\
\hline & Drilled water & $33 / 43(13)$ & 0.73 & {$[0.4-1.1]$} & 0.042 & & & \\
\hline & Other & $65 / 107(60)$ & 1 & - & - & & & NS \\
\hline \multirow[t]{2}{*}{ Eating raw fish } & Never/rarely & $235 / 384(61)$ & Ref & & & & & \\
\hline & Sometimes/usually/always & $14 / 16(88)$ & 4.4 & {$[0.9-19.8]$} & 0.021 & & & NS \\
\hline \multirow[t]{2}{*}{ Eating raw meat } & Never/rarely & $221 / 365(61)$ & Ref & & & & & \\
\hline & Sometimes/usually/always & $28 / 35(80)$ & 2.6 & {$[1.1-6.1]$} & 0.028 & & & NS \\
\hline \multirow[t]{2}{*}{ Eating snail } & Never/rarely & $234 / 383(61)$ & Ref & & & Ref & & \\
\hline & Sometimes/usually/always & $15 / 17(88)$ & 4.7 & {$[1.07-21.1]$} & 0.014 & 13.5 & [2.4-74.4] & 0.003 \\
\hline \multirow[t]{2}{*}{ Eating seafood } & Never & $134 / 209(64)$ & Ref & & & & & \\
\hline & $\begin{array}{l}\text { Rarely/sometimes/usually/ } \\
\text { always }\end{array}$ & $115 / 191(60)$ & 0.8 & {$[0.5-1.2]$} & 0.421 & & & NS \\
\hline Cooked food by themselves & Never/rarely & $94 / 171(55)$ & Ref & & & & & \\
\hline & Sometimes/usually/always & $155 / 229(68)$ & 1.7 & {$[1.1-2.5]$} & 0.009 & & & NS \\
\hline Share spoon & Never & $224 / 368(61)$ & Ref & & & & & \\
\hline & Rarely/usually/always & $25 / 32(78)$ & 2.2 & {$[0.9-5.4]$} & 0.04 & & & NS \\
\hline Share glasses & Never/rarely & $64 / 106(60)$ & Ref & & & & & \\
\hline & Usually/always & $185 / 294(63)$ & 1.1 & {$[07-1.7]$} & 0.64 & & & NS \\
\hline Wash hands before eating & Never/rarely/sometimes & $50 / 95(53)$ & Ref & & & & & \\
\hline & Always & $199 / 305(65)$ & 1.6 & {$[1.0-2.6]$} & 0.02 & & & NS \\
\hline Wash hands after toilet & Never/rarely/sometimes & $65 / 116(56)$ & Ref & & & & & \\
\hline & Always & $184 / 284(65)$ & 1.4 & {$[0.9-2.2]$} & 0.3 & & & NS \\
\hline Wash hands with soap & Never/rarely & 68/136 (50) & Ref & & & Ref & & \\
\hline & Usually/always & $181 / 264(69)$ & 2.1 & [1.4-3.3] & 0.0003 & 2.5 & [1.4-4.3] & 0.001 \\
\hline
\end{tabular}

All other variables were not significant at the bivariate level.

(OR $=6.2$ [1.1-45.8], whereas those older than 20 years had the following risk factors: ethnicity $\mathrm{OR}=18.03$ [2.35-138.1] and washing hands with soap $(\mathrm{OR}=3.59$ [1.78-7.22]).

The Lao-Tai ethnic group, representing $80 \%$ of the cohort, had the same risk factors as the whole group, whereas the non-Lao-Tai group was too small to identify distinct risk factors.

We compared the Xiengkhouang results with historical data from neighboring Thailand (between 1971 and 2014). In Thailand, the age of $50 \%$ seropositivity shifted from 4.5 years in $1971 / 1972$ to 42.0 years in 2014 , reflecting a decrease in childhood infections due to improved sanitation (data and analysis by Sa-nguanmoo et al. ${ }^{8}$ ). In Xiengkhouang, 50\% HAV seropositivity was reached at 12.1 years of age, corresponding roughly to the situation in Thailand almost 20 years ago (Figure 2).

Vientiane capital seroprevalence. Overall, $45.5 \%$ of 795 participants from Vientiane capital were HAV seropositive, with $0.6 \%$ borderline. Among 51 younger than 1 year attending a central hospital in Vientiane capital for unrelated illness, approximately $50 \%$ were anti-HAV positive probably because of persisting maternal antibodies in this age-group. Of 200 adolescents aged $11-20$ years, $7 \%$ were anti-HAV seropositive. Finally, adult healthcare workers $(n=140)$ and blood donors $(n=255)$ aged 20 to 50 years had high anti-HAV seroprevalence of $86.4 \%$ and $76.9 \%$, respectively (Table 1, 


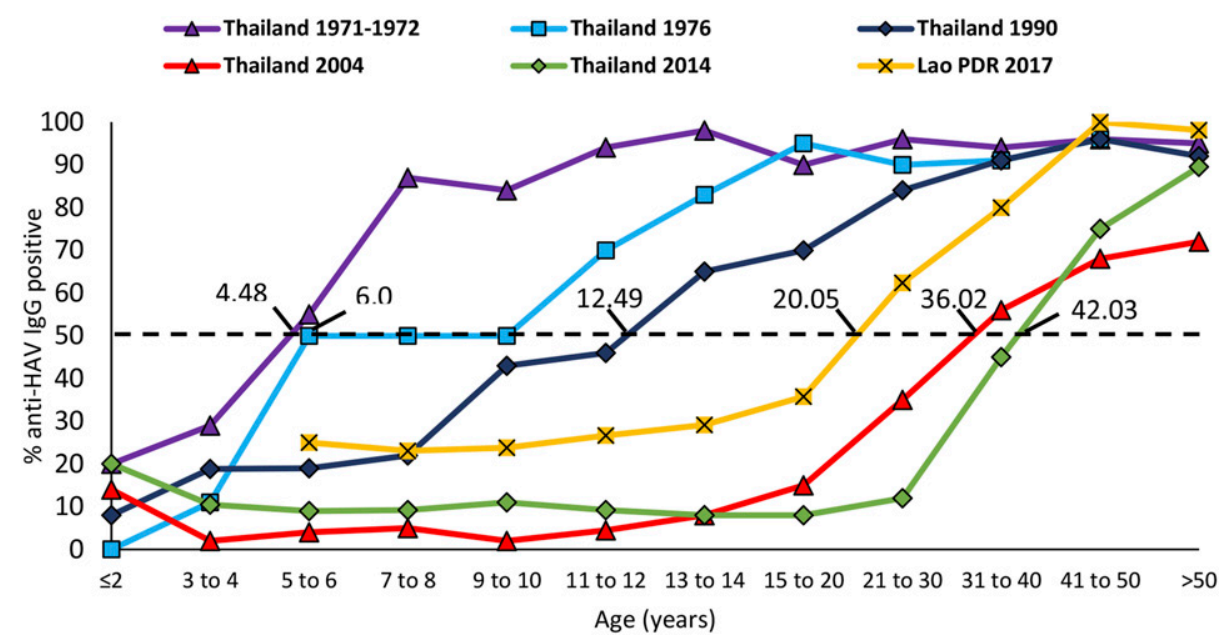

FIGURE 2. Age-stratified anti-hepatitis A virus IgG seroprevalence data from Xiengkhouang, Lao People's Democratic Republic, in 2017 compared with Thailand from 1971/1972 to 2014. Dotted line corresponds to the age of 50\% seropositivity. Adapted from Sa-nguanmoo et al. ${ }^{8}$ This $^{2}$ figure appears in color at www.ajtmh.org.

Figure 3). No risk factor data were available for the Vientiane cohorts.

\section{DISCUSSION}

In Xiengkhouang Province, almost two-thirds of the participants had previous infections with HAV. Already at the age of 5 to 10 years, $24 \%$ of children were HAV seropositive in this province. This early infection can only be explained by direct contact with unsafe water and food. ${ }^{12}$ As expected, the rate of past infections increased steadily with age. In the participants older than 30 years, more than $80 \%$ had previous HAV infection in both the rural setting of Xiengkhouang and the urban setting of Vientiane capital. There was also essentially no difference between the two adult cohorts in Vientiane (blood donors and healthcare workers). Even considering high rates of asymptomatic infections, particularly in children, the $<1,000$ cases reported during the past 5 years nationwide seems relatively low and suggestive of underreporting. In the capital, there was a conspicuous difference between cohorts born before and after 1996. This may reflect infections of these individuals during childhood and an improvement in sanitation in the late 1990s. Indeed, sanitation coverage in Lao PDR increased from $11 \%$ to $48 \%$ between 1990 and $2005 .{ }^{13}$ Similarly, in neighboring Thailand, improved sanitation resulted in a reduction in childhood infections and, therefore, a shift in the age distribution of anti-HAV positive individuals. The age at which $50 \%$ of the population is anti-HAV positive in Xiengkhouang is about 20 years, similar to that in Thailand between 1990 and 2004. . $14,15^{-15}$

Participants born after 1996 showed a seroprevalence below $10 \%$ in Vientiane capital but $20-25 \%$ higher in Xiengkhouang. However, by the age of 21-30 years, seroprevalence was similar in both settings. This suggests that the risk factors were higher in Xiengkhouang during the past 2 decades than the capital. Overall, $50 \%$ of children younger than 1 year had

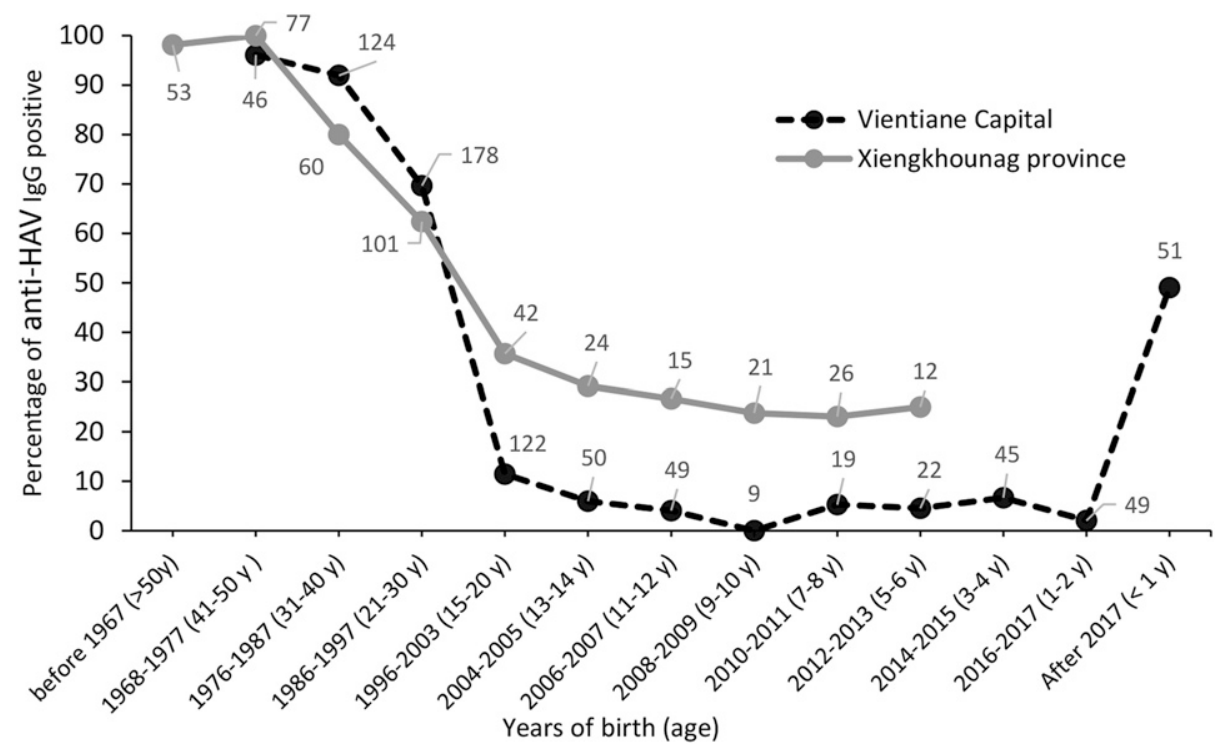

FIGURE 3. Comparison of anti-hepatitis A virus IgG positivity between Vientiane capital and Xiengkhouang Province. Numbers indicate the total number of participants per age-group. 
persisting maternal antibodies. This protective effect, in addition to breastfeeding, resulted in a low seroprevalence in the 1 - to 2-year-olds.

The risk practices were collected to determine the general sanitation and hygiene practices in the population, including factors related to HAV. Overall risk factors associated with anti-HAV IgG in Xiengkhouang Province were age, non-LaoTai ethnicity, frequently eating snails, and usually washing hands with soap. The most common sources of snails are rivers or rice fields. They are usually cooked before consumption and rarely eaten raw. Although "snails" have not previously been reported as a source of HAV infection, consumption of mollusks is a well-known risk factor. ${ }^{16-19}$ The non-Lao-Tai ethnicities in Lao PDR often have a lower socioeconomic status, poor housing and sanitation facilities, and less access to clean water, all explaining the higher HAV seroprevalence in this group. ${ }^{20,21}$ The result that washing hands with soap was slightly associated with an increased risk of anti-HAV was surprising and difficult to explain. The weak explanatory power of the final regression model is likely due to the high seroprevalence, reflecting a ubiquitous risk factor in the general environment. Furthermore, the IgG serology data relate to exposure in the past, whereas the risk practices concern current behavior. Therefore, associations with serology should be interpreted with caution.

A limitation of our study related to the fact that the sampling took place only in two provinces. Therefore, the data are not representative of other locations in Lao PDR, and we cannot extrapolate the data nationwide.

In summary, this study found significant exposure to HAV in Lao PDR. Most adult population has been exposed, probably during their early lives before general sanitation had improved. Currently, some exposure still begins early, particularly in rural areas with lesser sanitation, although progress has been made in Vientiane capital. As a consequence, the proportion of unexposed, susceptible adults is increasing, which may result in more frequent outbreaks in the older populations. In Lao PDR, compared with neighboring Thailand, the impact of improved sanitation on the reduction of HAV infections seems to have begun 20 years later. Improvements in water (and food) sanitation and better hygienic practices are still warranted, particularly in rural districts, to reduce the infection rate even further. The high HAV seroprevalence detected and the comparatively low numbers of reported cases in the past could indicate an underreporting of outbreaks. Improved reporting could provide guidance for identifying sources of infection, thereby mitigating exposure. We also recommend awareness campaigns to increase knowledge of the population regarding food- and sanitation-related infections. The lack of clean water sources should be addressed by investment in water, sanitation, and hygiene.

Received June 14, 2019. Accepted for publication February 25, 2020. Published online April 27, 2020.

Acknowledgments: We would like to thank Paul Brey and the local health officers for facilitating the study. We also wish to thank the participants for consenting to give samples for this research and the Institute de la Francophonie pour la Medecine Tropicale (IFMT) who collaborated with us.

Disclosure: This work was supported by the Luxembourg Ministry of Cooperation and the Luxembourg Institute of Health.
Authors' addresses: Vilaysone Khounvisith, Phonethipsavanh Nouanthong, Bounta Vongphachanh, and Antony P. Black, Lao-Lux Laboratory, Institut Pasteur du Laos, Vientiane, Lao PDR, E-mails: kvs.vilaysone@gmail.com, thip_mt@hotmail.com, b.vongphachanh@ pasteur.la, claude.muller@lih.lu, and a.black@pasteur.la. Xaipasong Xaiyaphet, Mahosot Hospital, Thanon Mahosot, Vientiane, Lao PDR, E-mail: namun_xyp@hotmail.com. Phetsavanh Chanthavilay, University of Health Sciences, Vientiane, Lao PDR, E-mail: phetsavanh456@ gmail.com. Daniel Reinharz, Laval University, Quebec, Canada, E-mail: daniel.reinharz@fmed.ulaval.ca.

\section{REFERENCES}

1. Wasley A, Fiore A, Bell BP, 2006. Hepatitis A in the era of vaccination. Epidemiol Rev 28: 101-111.

2. Jacobsen $\mathrm{KH}$, Wiersma ST, 2010. Hepatitis A virus seroprevalence by age and world region, 1990 and 2005. Vaccine 28: 6653-6657.

3. Jacobsen $\mathrm{KH}, 2010$. The global prevalence of hepatitis $A$ virus infection and susceptibility: a systematic review. Immunization, Vaccin Biol 1-428.

4. David AM, 2004. Hepatitis A outbreaks - methods of intervention in south-east Asian countries. Int J Infect Dis 8: 201-209.

5. Public Health England, 2014. UK standards for microbiology investigation: hepatitis A virus acute infection serology. Virology 27: 1-13.

6. World Health Organization, 2012. WHO position paper on hepatitis A vaccines-June 2012. Weekly Epidemiological Record 87: 261-276.

7. SAGE Hepatitis A Working Group, 2011. Evidence Based Recommendations for Use of Hepatitis A Vaccines in Immunization Services: Background Paper for SAGE Discussions. Geneva, Switzerland: World Health Organization.

8. Sa-nguanmoo $P$ et al., 2016. Declining trend of hepatitis $A$ seroepidemiology in association with improved public health and economic status of Thailand. PLoS One 11: e0151304.

9. Franco E, Meleleo C, Serino L, Sorbara D, Zaratti L, 2012. Hepatitis A: epidemiology and prevention in developing countries. World J Hepatol 4: 68-73.

10. Provicial Department Natural Resources and Environment, 2013. Provincial Biodiversity Strategy and Action Plan 2012-2020 Xieng Khouang Province Lao PDR. Vientiane, Lao PDR: Lao Ministry of Health.

11. Pourhoseingholi MA, Vahedi M, Rahimzadeh $M, 2006$. Sample size calculation in epidemiological studies. Theory Pract 7: 225-231.

12. Gall AM, Marinas BJ, Lu Y, Shisler J, 2015. Waterborne viruses: a barrier to safe drinking water. PLoS Pathog 11: e1004867.

13. World Bank, 2009. Economic Impacts of Sanitation in Lao PDR. Washington, DC: The World Bank.

14. Barzaga NG, 2000. Hepatitis A shifting epidemiology in southeast Asia and China. Vaccine 18 (Suppl 1): S61-S64.

15. Moisseeva AV, Marichev IL, Biloschitchkay NA, Pavlenko KI, Novik LV, Kovinko LV, Lyabis OI, Houillon G, Rasuli A, 2008. Hepatitis A seroprevalence in children and adults in Kiev city, Ukraine. J Viral Hepat 15: 43-46.

16. Seo $\mathrm{J}$ et al., 2013. Risk factors for acute hepatitis a infection in Korea in 2007 and 2009: a case-control study. J Korean Med Sci 28: 908-914.

17. Koopmans M, Duizer E, 2004. Foodborne viruses: an emerging problem. Int J Food Microbiol 90: 23-41.

18. Roldan EM, Rodrıguez EE, Garcia ME, Navajas M, 2013. Prevalence of hepatitis A virus in bivalve molluscs sold in granada (Spain) fish markets. Foodborne Pathog Dis 10: 528-532.

19. laconelli $M$ et al., 2015. Hepatitis $A$ and $E$ viruses in wastewaters, in river waters, and in bivalve molluscs in Italy. Food Environ Virol 7: 316-324.

20. Engvall A, 2006. Ethnic Minorities and Rural Poverty in Lao PDR. Stockholm School of Economics, The European Institute of Japanese Studies.

21. Andersson M, Engvall A, Kokko A, 2006. Determinants of Poverty in Lao PDR. Stockholm School of Economics. Available at: http://lup.lub.lu.se/record/3127811. 